\title{
miR-503 inhibits proliferation making human hepatocellular carcinoma cells susceptible to 5-fluorouracil by targeting EIF4E
}

\author{
XIAOYAN YANG ${ }^{1,2}$, JINGLEI ZANG $^{4}$, XIA PAN $^{1}$, JIE YIN $^{1}$, QIONG XIANG $^{1}$, \\ JIA YU $^{1}$, RUNLIANG GAN ${ }^{2,3}$ and XIAOYONG LEI ${ }^{1,2}$ \\ ${ }^{1}$ Institute of Pharmacy and Pharmacology, ${ }^{2}$ Hunan Province Cooperative Innovation Center for \\ Molecular Target New Drug Study, ${ }^{3}$ Institute of Tumor Research, University of South China, Hengyang, Hunan 421001; \\ ${ }^{4}$ Changsha Health Vocational College, Changsha, Hunan 410100, P.R. China
}

Received June 5, 2016; Accepted October 31, 2016

DOI: $10.3892 /$ or.2016.5220

\begin{abstract}
Hepatocellular carcinoma (HCC), a disease that is a major health care issue across the globe, includes the deviant expression of miRNAs in its development, progression, and resistance to treatment. We focused our study on miR-503 expression and its role in HCC. miR-503 was found in HCC tissues and cell lines using quantitative real-time PCR (RT-qPCR). Western blot analyses and the luciferase reporter assay were used to determine the miR-503 potential target in the HCC cells. We used MTT to analyze cell proliferation activity and noted that there was a considerable decrease of miR-503 in HCC tissues and cell lines when measured against the controls. miR-503 upregulation decreased expression of eukaryotic translation initiation factor 4E (EIF4E), and reduced HCC cell proliferation and sensitized HCC cells to anticancer drugs. miR-503 overexpression hindered luciferase activity of EIF4E 3' untranslated region-based reporter construct among HepG2, BEL-7402, and SMMC-7721 cells, revealing that miR-503 may increase sensitivity to therapies at least partially through targeting EIF4E suppression of HCC proliferation.
\end{abstract}

Correspondence to: Professor Xiaoyong Lei, Hunan Province Cooperative Innovation Center for Molecular Target New Drug Study, University of South China, 28 Changsheng Road, Hengyang, Hunan 421001, P.R. China

E-mail: 1622214323@qq.com

Professor Runliang Gan, Institute of Tumor Research, University of South China, 28 Changsheng Road, Hengyang, Hunan 421001, P.R. China

E-mail: ganrunliang@163.com

Abbrevations: EIF4E, eukaryotic initiation factor 4E; miRNA, microRNA; MTT, 3-(4,5-dimethylthiazol-2-yl)-2,5-diphenyltetrazolium bromide; qRT-PCR, quantitative real-time reverse transcriptionpolymerase chain reaction

Key words: miR-503, eukaryotic translation initiation factor 4E, 5-fluorouracil, drug sensitivity, hepatocellular carcinoma cells

\section{Introduction}

Patients with hepatocellular carcinoma (HCC), one of the most prevalent types of malignancies that is the third most common type of cancer deaths across the globe (1), are frequently administered platinum-based chemotherapy, most likely combined with radiation treatments. Unfortunately, intrinsic or acquired chemoresistance is a large issue in producing a successful outcome for the patient (2). Prior studies have revealed the cytological mechanisms of therapy resistance in cancer cells. MicroRNAs (miRNAs) are internal, noncoding, $\sim 22$ nt small RNAs that display cell- and tissue-specific expression. They play important-regulatory roles in cell apoptosis, proliferation and development. Their divergent expression has been proven to be involved in therapy resistance in varying kinds of tumors (3-6). miRNAs activate mRNA degradation or translational repression because they bind to corresponding sequences of the targeted mRNAs (7).

Researchers have shown heightened enthusiasm aimed at comprehending the role of miR-503 in cancer. miR-503, an intragenic miRNA found on the Xq26.3 chromosome, was first established in human retinoblastoma tissues with microRNA microarray technology $(8,9)$. Divergent expression of miR-503 and its human oncology role are newly documented. For example, Peng et al (10) found that miR-503 expression is diminished in gastric cancer cell lines and that miR-503 prevents gastric cancer cell proliferation, migration and invasion. Chong et al (11) noted that miR-503 was downregulated in osteosarcoma cell lines and primary tumors, and that restoration of miR-503 lowered cell proliferation, migration and invasion. Zhang et al (12) demonstrated that miR-503 expression was significantly lowered in glioblastoma multiforme tissues and cell lines, while miR-503 overexpression limited cell proliferation by prompting apoptosis with the targeting of IGF-1R. Wang et al (13) reported that miR-503 controls cisplatin resistance of human gastric cancer cell lines with the targeting of IGF-1R and Bcl-2. Unfortunately, only a sparse amount has been verified regarding the effects of miR-503 on the evolution of therapy resistance in HCC patients.

The proto-oncogene eukaryotic translation initiation factor 4E (EIF4E), is the control; overexpression of EIF4E 
precisely elevates the mRNA translation of proteins that are linked with tumor growth, invasion and metastasis. EIF4E overexpression has also been discovered in different malignant tumors, including liver, cervical, ovarian, esophageal and lung cancer. Prior evaluations have demonstrated that EIF4E is overexpressed in $\sim 100 \%$ of head and neck cancers and has been connected with poor prognosis (14-16).

We initially analyzed miR-503 expression in HCC tissues and cell lines in comparison to normal controls and we then focused on miR-503 expression in HCC and its effect on the proliferation and resistant to therapies. Then we explored the target effect on the HCC cell drug resistance and established EIF4E as one of its direct targets in HCC cells. Thus, our data display the pivotal role miR-503 plays in HCC pathogenesis and suggests its potential in cancer treatment.

\section{Methods and materials}

Materials and tissue collection. The HCC cell lines HepG2, BEL-7402, SMMC-7721, and human normal liver cell line L-02 were obtained from the Cell Bank of the Chinese Academy of Sciences (Shanghai, China). The DMEM, RPMI-1640 medium, and fetal bovine serum were from HyClone Laboratories (Beijing, China) and miR-503 mimic and siEIF4E were from GenePharma Co., Ltd. (Shanghai, China). HCC tissues and adjacent normal liver tissues were obtained from patients who had undergone surgery at the Second Affiliated Hospital of University of South China, between 2014 and 2015 and were diagnosed with HCC after histopathological evaluation. No local or systemic preoperative treatment had been performed on the patients. Tissue samples were collected, snap frozen in liquid nitrogen, and stored at $-80^{\circ} \mathrm{C}$ until RNA was extracted. The study was approved by the Research Ethics Committee of University of South China (Hunan, China) and informed consent was granted by the patients.

Cell culture and transfection. HCC cell lines, BEL-7402, and SMMC-7721 were cultured in RPMI-1640 medium, while HepG2 and the human normal liver cell line L-02 were cultured in DMEM medium supplemented with $10 \%$ fetal calf serum in a humidified atmosphere containing $5 \% \mathrm{CO}_{2}$ at $37^{\circ} \mathrm{C}$. We purchased hsa-miRNA-503 mimic and negative control from GenePharma Co., Ltd. For convenience, hsa-miRNA-503 mimic and negative control were referenced as miR-503 mimic and negative control, respectively. Cells were cultured for a minimum of $24 \mathrm{~h}$ before transfection in complete medium minus antibiotics. Cells were washed with PBS (pH 7.4) and then transiently transfected with $50 \mathrm{nM}$ miR-503 mimic or negative control, using Lipofectamine ${ }^{\mathrm{TM}} 2000$ (Invitrogen, Carlsbad, CA, USA) according to the manufacturer's instructions.

Transfection of siRNA. EIF4E siRNA was purchased from GenePharma Co.,Ltd. Cells were plated in antibiotic-free growth medium at $40-50 \%$ confluence $\sim 24 \mathrm{~h}$ before transfection. RNA oligonucleotides were transfected using Lipofectamine ${ }^{\mathrm{TM}} 2000$ (Invitrogen) according to the company protocol.

$R T$ - $q P C R$. RT-qPCR was performed and total RNAs were extracted from cells using the TRIzol reagent (Tiangen, Beijing,
China) according to the manufacturer's instructions. Primer sequences of EIF4E, GAPDH, miR-503, U6 were: i) EIF4E forward, 5'-ACGGAATCTAATCAGGAGGT-3' and reverse, 5'-TTCCCACATAGGCTCAATA-3'; ii) GAPDH forward, 5'-CATGAGAAGTATGACAACAGCCT-3' and reverse, 5'-AGT CCTTCCACGATACCAAAGT-3'; iii) miR-503 forward, 5'-CGCGGGATCGGGTCAGA-3' and reverse, 5'-GGGAAC ATGTTGATCTCAG-3'; and iv) U6 snRNA forward, 5'-ATT GGAACGATACAGAGAAGATT-3' and reverse, 5'-GGAACGC TTCACGAATTTG-3'. The level of U6 snRNA was used as internal control for miR-503 quantification. Total RNA was reverse transcribed to cDNA using the custom reverse transcription kit; and qPCR analyses of EIF4E were performed using the above primer and normalized to GADPH to determine the EIF4E mRNA level. Specifically, SYBR-Green qPCR was conducted on an Mx3000P Real-Time PCR system (Stratagene, Santa Clara, CA, USA). The cycling program consisted of 40 cycles (each for $3 \mathrm{~min}$ at $95^{\circ} \mathrm{C}, 12 \mathrm{sec}$ at $95^{\circ} \mathrm{C}$ and $40 \mathrm{sec}$ at $62^{\circ} \mathrm{C}$ ). The relative expression ratios of EIF4E and miR-503 were determined with the $2^{-\Delta \Delta \mathrm{Ct}}$ method.

Western blotting. Either the miR-503 mimic or miR NC was used to transfect the cells. At $48 \mathrm{~h}$ post-transfection, total protein was removed from the HepG2, BEL-7402 and SMMC-7721 cells and theirs untransfected cells as well as human normal liver cells L-02 with the RIPA cell lysis reagent containing proteinase and phosphatase inhibitors (Sangon Biotech, Shanghai, China) at $4^{\circ} \mathrm{C}$ for $30 \mathrm{~min}$. Cell lysates were centrifuged $\left(13,000 \times \mathrm{g}\right.$ for $20 \mathrm{~min}$ at $\left.4^{\circ} \mathrm{C}\right)$, and protein concentrations of the supernatant were determined with the BCA protein assay reagent kit (Thermo Fisher Scientific Inc., Waltham, MA, USA). Supernatants containing total protein were combined with a correlating volume of 5X SDS loading buffer and heated at $100^{\circ} \mathrm{C}$ for $10 \mathrm{~min}$. Then, the supernatant lysates were run on $10 \%$ SDS-polyacrylamide gels (50 $\mu \mathrm{g} / \mathrm{lane})$ and proteins were moved to poly(vinylidene fluoride) (PVDF) membranes (Bio-Rad Laboratories, Hertfordshire, UK) by semidry electroblotting $\left(1.5 \mathrm{~mA} / \mathrm{cm}^{2}\right)$. A PageRuler prestained protein ladder (CWBio) was implemented as a molecular marker. A sole membrane was divided into three parts at the 30 and $45 \mathrm{kDa}$ bands, and incubated with anti-EIF4E (1:500; rabbit no. 33766) monoclonal rabbit primary antibodies (Abcam, Cambridge, MA, USA) and anti- $\beta$-actin $(1: 1,000$; rabbit no. 4970) monoclonal rabbit primary antibodies (Cell Signaling Technology, Danvers, MA, USA), respectively. Proteins were located with horseradish peroxidase-conjugated goat anti-rabbit IgG secondary antibody (1:4,000; rabbit no. CW0103; CWBio) and a ChemiLucent ECL detection system (EMD Millipore, Billerica, MA, USA). ImageJ software (National Institutes of Health, Bethesda, MD, USA) was used to quantify the protein bands.

MTT analysis. The HepG2, BEL-7402 and SMMC-7721 cells in the exponential growth phase were seeded into 96-well plates with a density of $4 \times 10^{3}$ cells/well and incubated for $12 \mathrm{~h}$ at $37^{\circ} \mathrm{C}$, respectively. Cells were transfected with either the miR-503 mimic or miR NC. At $24 \mathrm{~h}$ post-transfection, various concentrations of 5-fluorouracil (5-FU) $(0,0.1,1,10$ and $100 \mu \mathrm{M})$ were added. Subsequent to incubation for $24 \mathrm{~h}, 20 \mu \mathrm{l}$ MTT (Amresco LLC, Solon, OH, USA) solution, concentrated 
A

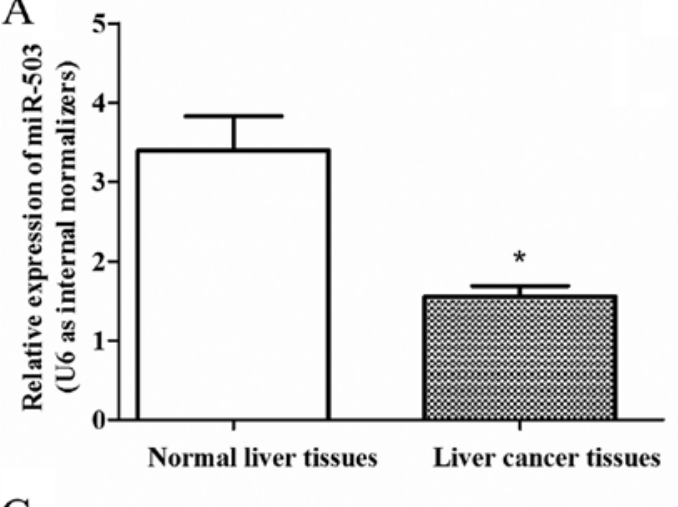

$\mathrm{C}$

EIF4E

$\beta$-actin

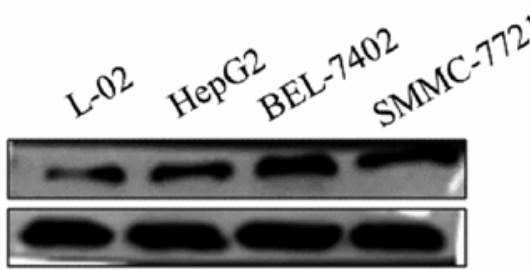

$\mathrm{E}$

$\frac{\text { HepG2 }}{\text { Blank } \begin{array}{c}\text { Negative } \\ \text { control miR-503 } \\ \text { mimic }\end{array}}$

EIF4E

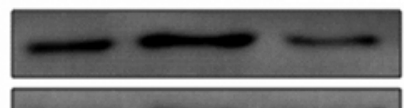

$\beta$-actin

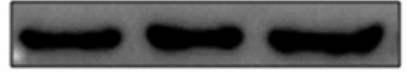

BEL-7402

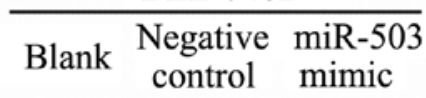

EIF4E

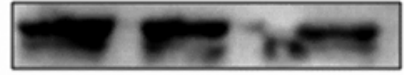

$\beta$-actin

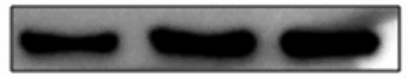

SMMC-7721

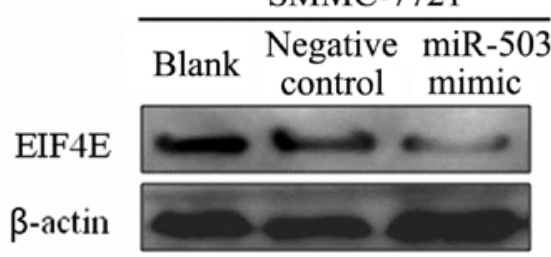

B

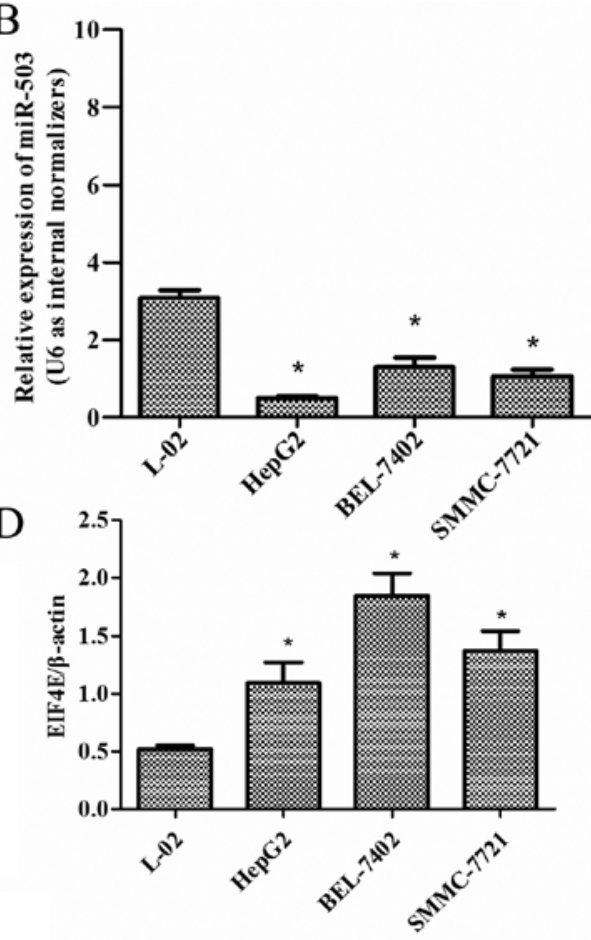

F
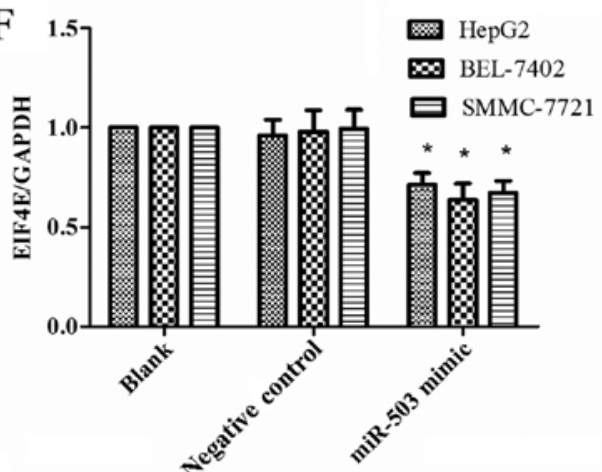

G

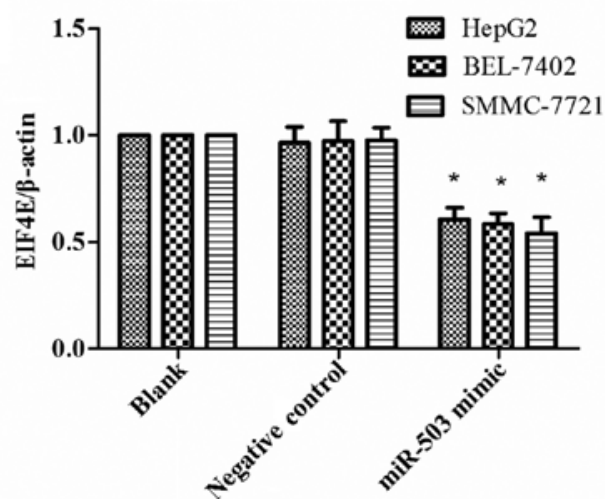

Figure 1. miR-503 is downregulated in HCC tissue and HCC cell lines, miR-503 and EIF4E levels were negatively correlated. (A) miR-503 is significantly decreased in human hepatocellular carcinoma tissues in comparison to adjacent-normal liver cancer tissues. * ${ }^{*}<0.05$ compared to adjacent-normal liver cancer tissues. (B) The expression level of miR-503 in three HCC cell lines and normal L-02 cells. (C and D) We detected the expression of EIF4E among L-02, HepG2, BEL-7402 and SMMC-7721 cells. EIF4E protein highly expressed in HepG2, BEL-7402 and SMMC-7721 cells compared to L-02 cells. (E) Expression levels of EIF4E mRNA and (F and G) protein in the miR-503 mimic-transfected HepG2, BEL-7402 and SMMC-7721 cells. After transfection, relative EIF4E mRNA and protein levels were downregulated significantly, they were normalized to GAPDH or $\beta$-actin, respectively. "p<0.05 compared to control cells and untreated cells. Assays were performed in triplicate. Means \pm SD is shown.

at $5 \mathrm{mg} / \mathrm{ml}$, was placed in each well for $4 \mathrm{~h}$ at $37^{\circ} \mathrm{C}$. Culture medium was removed, and the insoluble formazan crystals were dissolved in $150 \mu \mathrm{l}$ dimethyl sulfoxide (Beijing Dingguo Changsheng Biotechnology Co.,Ltd., Beijing, China). Following agitation for $10 \mathrm{~min}$, the absorbance at $490 \mathrm{~nm}$ (A490) was optically monitored with a 318-microplate reader (Shanghai Sanco Instrument Co., Ltd., Shanghai, China).

Target prediction. TargetScan (http://www.targetscan. org), microRNA.org (http://www.microrna.org/) and Pictar 

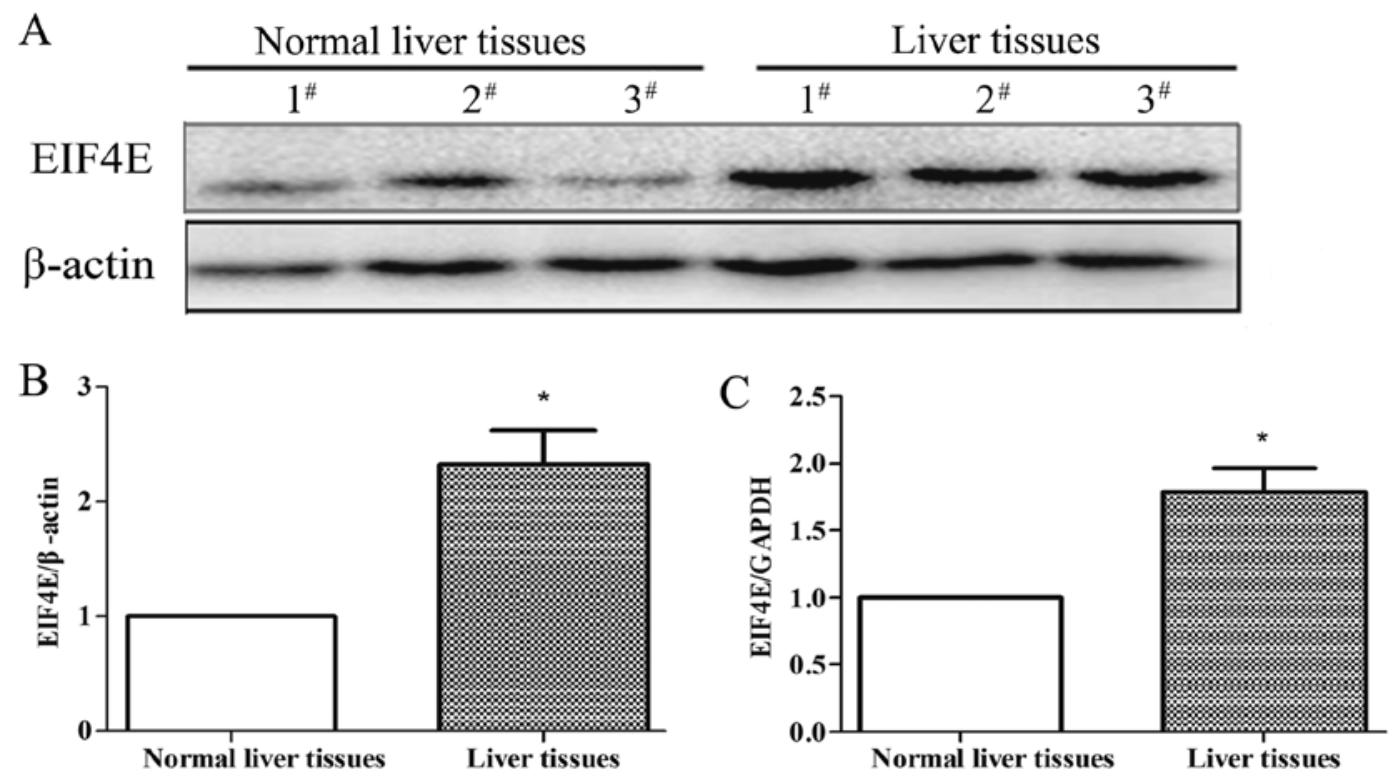

Figure 2. Expression of EIF4E is upregulated in human hepatocellular carcinoma tissues. (A and B) Western blotting of EIF4E protein, (C) qRT-PCR of EIF4E mRNA in human hepatocellular carcinoma tissues and adjacent-normal liver cancer tissues. " $\mathrm{p}<0.05$ compared to adjacent-normal liver cancer tissues. Assays were performed in triplicate. Means $\pm \mathrm{SD}$ is shown.

A

$\frac{\text { HepG2 }}{\begin{array}{c}\text { Negative } \\ \text { control siEIF4E }\end{array}}$

EIF4E

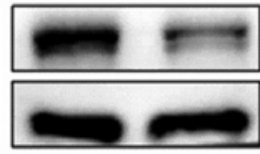

$\beta$-actin
BEL-7402

Negative
control siEIF4E

EIF4E

$\beta$-actin

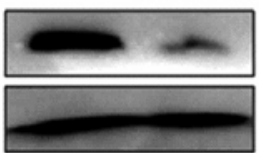

EIF4E

SMMC-7721

$\mathrm{B}$

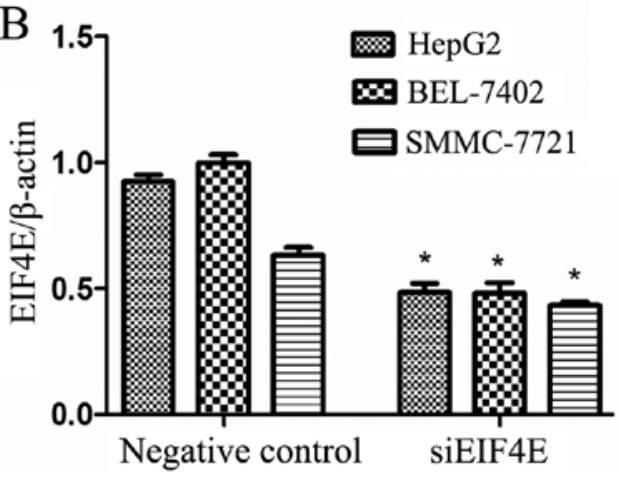

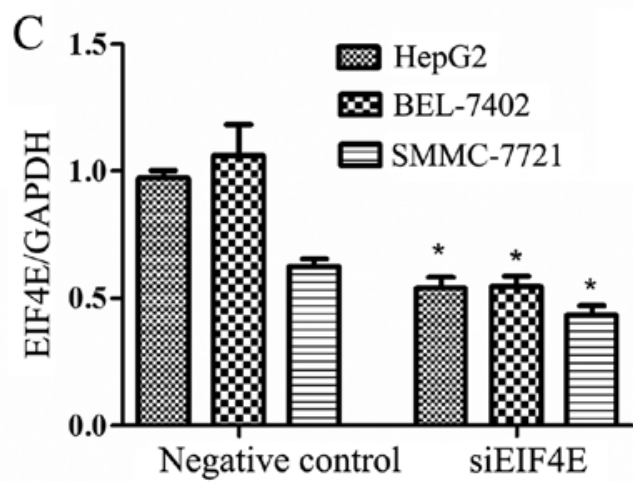

Figure 3. Anticancer efficacy of the silencing of EIF4E expression. (A and B) Western blotting of EIF4E protein and (C) qRT-PCR of EIF4E mRNA in siRNA EIF4E or negative control treated HepG2, BEL-7402 and SMMC-7721 cells. * p<0.05, compared to control cells. Assays were performed in triplicate. Means \pm SD is shown.

(http://pictar.mdc-berlin.de/cgi-bin/PicTar_vertebrate.cgi) algorithms determined the putative targeted genes of miR-503.

Construction of EIF4E 3'-UTR-luciferase plasmids and reporter assays. The 3'-untranslated region (3'-UTR) of human EIF4E was amplified from human genomic DNA and singly inserted into the psiCHECK ${ }^{\mathrm{TM}}-2$ vector psiCHECK ${ }^{\mathrm{TM}}-2$ (Land, Guangzhou, China) with the XhoI and NotI sites. Likewise, a portion of the EIF4E 3'-UTR mutant was placed into the psiCHECK ${ }^{\mathrm{TM}}-2$ control vector at the same locations. To create reporter assays, the wild-type and mutant EIF4E 3'-UTR-luciferase reporter plasmids were co-transfected with miR-503 mimic or miR NC (GenePharma Co., Ltd.) into HepG2, BEL-7402 and SMMC-7721 cells (American Type Culture Collection, Manassas, VA, USA) with Lipofectamine 2000 (Invitrogen Life Technologies), respectively. At $48 \mathrm{~h}$ post-transfection, the cells were assayed for luciferase activity using a Dual-Luciferase assay system (Promega Corporation, Madison, WI, USA) according to the manufacturer's instructions. The Renilla luciferase activities were normalized to the corresponding activities of firefly luciferase. For each transfection, the average luciferase activity was calculated from three replicates. 

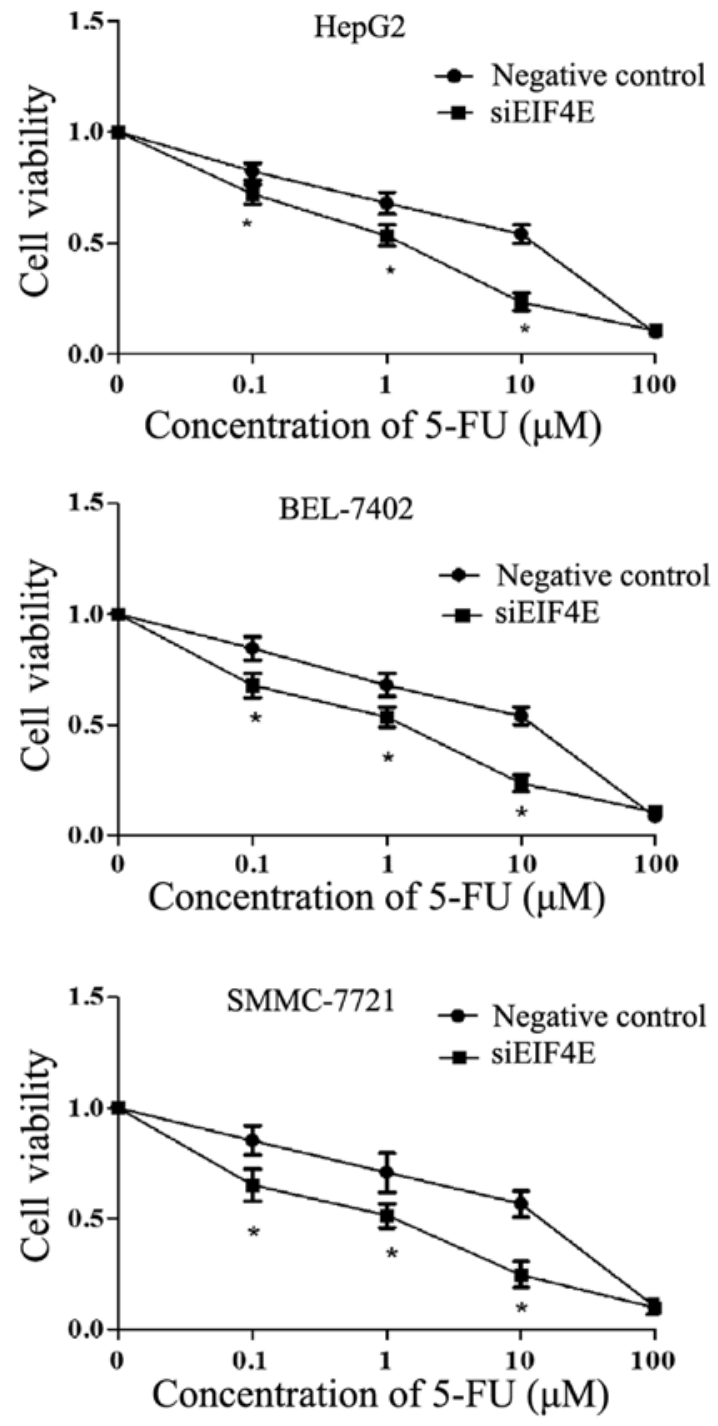

Figure 4. Effects of EIF4E on the sensitivity of HepG2, BEL-7402 and SMMC-7721 cells to 5-FU. siRNA EIF4E or negative control treated HepG2, BEL-7402 and SMMC-7721 cells were tested by increasing concentrations of the tested drug and measuring the surviving cells with the MTT cell proliferation assay at $48 \mathrm{~h}$ after the cells were treated, respectively. siEIF4E transfection substantially lowered cell viability when contrasted with the negative control. " $\mathrm{p}<0.05$ compared to control cells. Assays were performed in triplicate. Means \pm SD is shown.

Statistical analysis. SPSS software, version 18.0 (SPSS, Inc., Chicago, IL, USA) was used for statistical analysis. Data are expressed as mean $\pm \mathrm{SD}$ and analyzed using one-way analysis of variance (ANOVA) and least significant difference (LSD) tests. Statistical significance was set as $\mathrm{p}<0.05$.

\section{Results}

miR-503 is downregulated in HCC tissue and cell lines, miR-503 and EIF4E levels are negatively correlated. To evaluate if miR-503 is downregulated in HCC, we determined the mature miR-503 level in HCC and pair-matched adjacent healthy liver tissues by RT-qPCR using U6 that had not been deregulated in HCC to standardize. Our evaluation revealed that miR-503 tumor expression was substantially $(\mathrm{p}<0.05)$ lowered in 9 HCC samples based on the affiliated controls
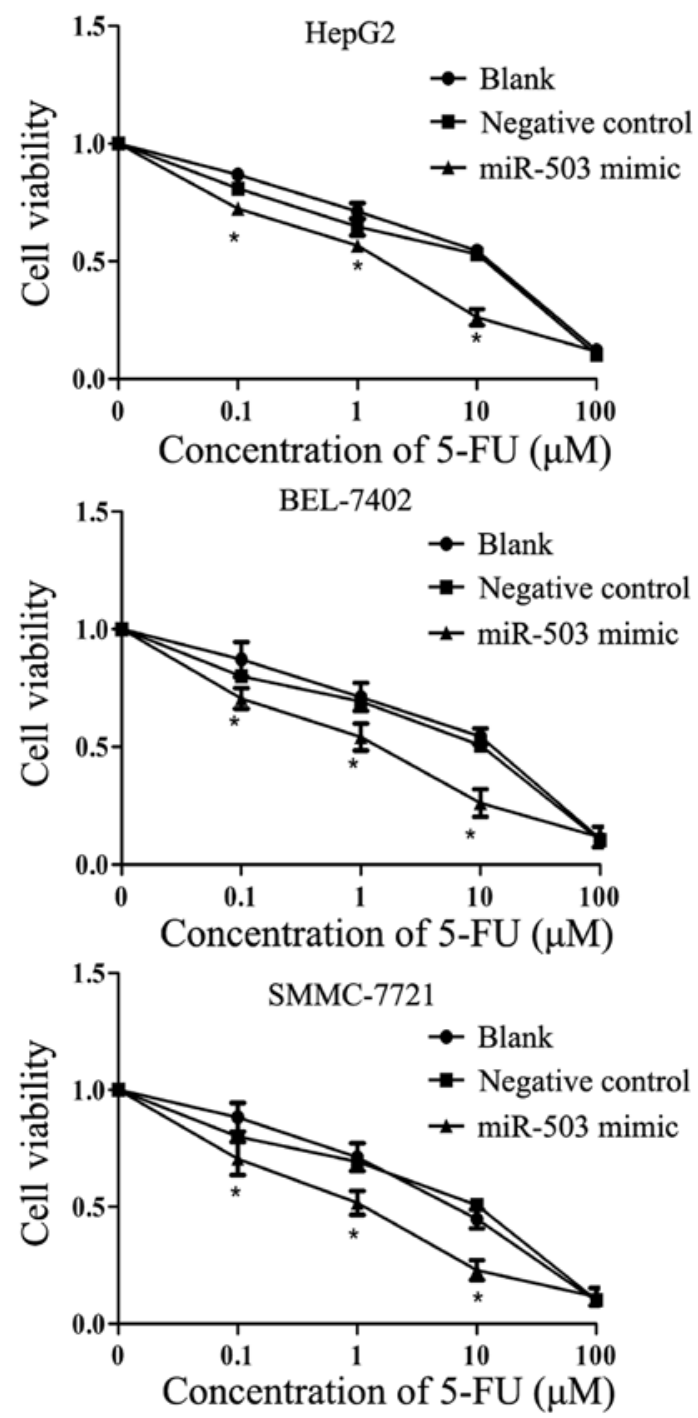

Figure 5. Effects of miR-503 on the sensitivity of HepG2, BEL-7402 and SMMC-7721 cells to 5-FU. Drug resistance of miR-503 mimic transfected cells, NC transfected cells and untreated cells were tested by increasing concentrations of the tested drug and measuring the surviving cells with the MTT cell proliferation assay at $48 \mathrm{~h}$ after the cells were treated, respectively. The outcome demonstrated that the transfection of miR-503 mimic substantially decreased cell viability in contrast to negative control and blank (untreated cells). ${ }^{*} \mathrm{p}<0.05$ compared to control cells and untreated cells. Assays were performed in triplicate. Means \pm SD is shown.

with the 9 evaluated samples (Fig. 1A). We then looked at miR-503 expression in HCC cell lines; we noted less miR-503 expression in HepG2, BEL-7402 and SMMC-7721 cell lines, in contrast to normal liver cells L-02 (Fig. 1B). Additionally, these results demonstrated that the lowered miR-503 expression may potentially be critical in the development and advancement of HCC. We used western blot analysis to detect the expression of EIF4E among L-02, HepG2, BEL-7402 and SMMC-7721 cells. The data showed that EIF4E protein was highly expressed in HepG2, BEL-7402 and SMMC-7721 cells compared to L-02 cells (Fig. 1C and D).

miR-503 has been noted to suppress tumors in testicular germ cell tumors. Next, we investigated whether miR-503 could play a role in regulating EIF4E expression. We transfected HepG2, BEL-7402 and SMMC-7721 cells with either miR-503 mimic or negative control, respectively, and 
A

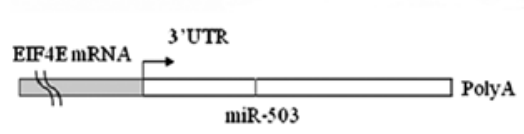

Conserved

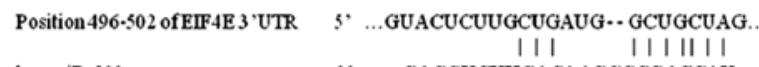

hsa-miR-503
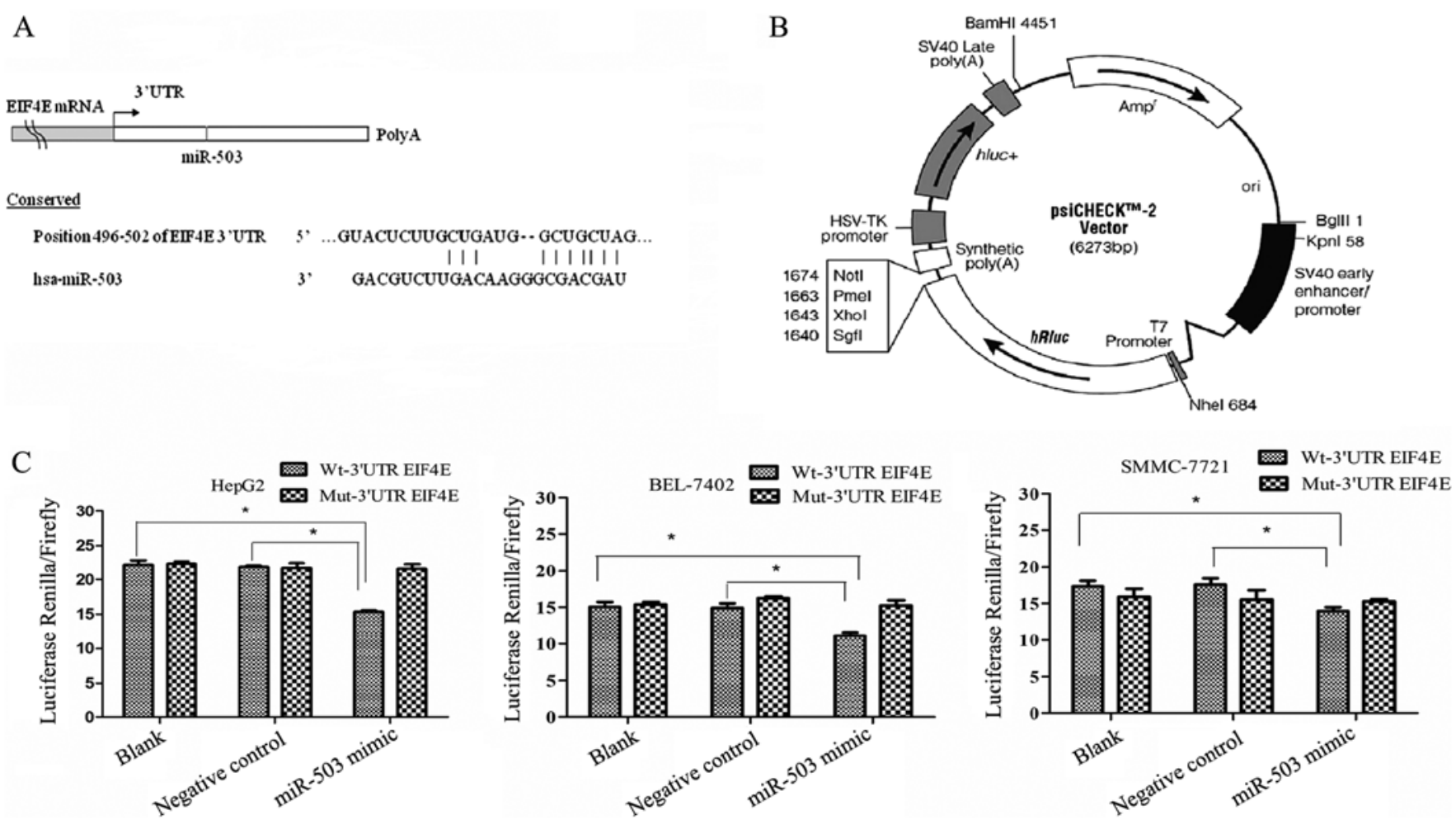

Figure 6. EIF4E is a target of miR-503 at specific 3'-UTR sites. (A) The 3'-UTR of EIF4E harbors miR-503 cognate sites. (B) psiCHECK ${ }^{\mathrm{TM}}$-2 dual-luciferase reporter vector. (C) Relative luciferase activity of reporter plasmids carrying wild-type or mutant EIF4E 3'-UTR in HepG2, BEL-7402 and SMMC-7721 cells co-transfected with miR-503 mimic or negative control. The outcome shows that miR-503 limits luciferase activity among HepG2, BEL-7402, and SMMC-7721 cells when the reporter plasmid transported the wild-type EIF4E 3'-UTR, but no substantial limitaion was noted when the reporter plasmid displayed a mutant EIF4E 3'-UTR. " p<0.05 compared to control cells and untreated cells. Assays were performed in triplicate. Means \pm SD is shown.

quantitated by real-time PCR. The miR-503 substantially downregulated EIF4E mRNA expression (Fig. 1E). Western blot results showed that enhanced expression of miR-503 significantly lowered the amount of EIF4E protein compared to blank and negative controls (Fig. $1 \mathrm{~F}$ and $\mathrm{G}$ ).

EIF4E expression is upregulated in HCC tissue. The proto-oncogene EIF4E is vital in controlling translation; overexpression precisely elevates the mRNA translation of proteins linked with tumor growth, invasion and metastasis, and prevents cell apoptosis in various tumors, including HCC. Thus, we evaluated EIF4E expression in human primary liver cancer and pair-matched adjacent liver tissues. The western blot results revealed that EIF4E protein expression was boosted in liver cancer tissues in contrast to normal liver tissues (Fig. 2A and B). This was verified with RT-qPCR of EIF4E mRNA expression (Fig. 2C).

Anticancer efficacy of silence of EIF4E expression combined with 5-FU in vitro. We next examined the potential tumorigenicity of EIF4E in HCC. The silence of EIF4E expression by siRNA significantly downregulated EIF4E protein (Fig. 3A and B) and mRNA expression (Fig. 3C). To further investigate whether EIF4E related to sensitivity of HepG2, BEL-7402 and SMMC-7721 cells to 5-FU, we transfected siEIF4E or negative control into HepG2, BEL-7402 and SMMC-7721 cells. The MTT cell proliferation assay was used to establish the cell survival rate. The result showed that siEIF4E transfection substantially lowered cell viability when contrasted with the negative control (Fig. 4). Thus, we adopted EIF4E as target for oncogenes.

miR-503 sensitizes HCC cells to 5-FU. miR-503 sensitized HepG2, BEL-7402 and SMMC-7721 cells to 5-FU. To further investigate whether miR-503 sensitized HepG2, BEL-7402 and SMMC-7721 cells to 5-FU, we transfected miR-503 mimic or negative control into HepG2, BEL-7402 and SMMC-7721 cells. The MTT cell proliferation assay was performed to determine cell survival rate. The outcome demonstrated that the transfection of miR-503 mimic substantially decreased cell viability in contrast to negative control and blank. These results show that miR-503 could sensitize HepG2, BEL-7402 and SMMC-7721 cells to 5-FU through regulated EIF4E expression (Fig. 5).

miR-503 targets human EIF4E. We then examined the undisclosed molecular mechanism of the antitumorigenic trait of miR-503 in HCC cells. Because the biological roles of miRNAs mainly intervene in animal cells by preventing the expression of target genes, we queried various databases (TargetScan, microRNA.org and PicTar) for possible targets displaying oncogenic characteristics. EIF4E, which protects the miR-503 related site, specifically, 496-502 of EIF4E 3'-UTR, is a predicted target of miR-503 (Fig. 6A). To evaluate if EIF4E expression is controlled by miR-503, the EIF4E 3'-UTR were replicated in a luciferase reporter plasmid, and the proficiency of miR-503 to prevent expression in the adjacent hRluc coding region was quantified. To do this, the luciferase reporter plasmid psiCHECK ${ }^{\mathrm{TM}}$-2-EIF4E-3'-UTR (Fig. 6B) or 
a mutant reporter plasmid with point mutations in the putative miR-503 binding sites was co-transfected with miR-503 mimic or negative control, individually. The outcome shows that miR-503 limits luciferase activity among HepG2, BEL-7402 and SMMC-7721 cells when the reporter plasmid transported the wild-type EIF4E 3'-UTR, but no substantial limitation was noted when the reporter plasmid displayed a mutant EIF4E 3'-UTR (psiCHECK ${ }^{\mathrm{TM}}$-2-mut-EIF4E -3'-UTR) (Fig. 6C), demonstrating that miR-503 fastened immediately to the proposed binding area in the EIF4E 3'-UTR.

\section{Discussion}

The present study revealed the following novel findings: i) exogenously overexpressed miR-503 inhibits human hepatocellular carcinoma cell proliferation and sensitizes cells to 5-FU; ii) silenced EIF4E expression inhibits human hepatocellular carcinoma cell proliferation and sensitizes cells to 5-FU; iii) miR-503 targets EIF4E in human hepatocellular carcinoma cells and negatively expressed with EIF4E; iv) our data showed that miR-503 may increase sensitivity to therapies through targeting EIF4E.

To date, platinum-based drugs, including 5-FU, in particular, are the major clinical pharmaceuticals for hepatocellular carcinoma. Despite intense attempts 5-FU administration frequently ends up with the patient developing a resistance to the pharmaceutical, which, in turn, concludes in therapy failure. Therefore, the resistance to 5-FU is a crucial clinical component that needs to be addressed and greater knowledge of the molecular mechanisms involved in drug resistance will assist in developing new drug targets and methods for eradicating human hepatocellular carcinoma. Although dysregulation of miRNAs has demonstrated its participation in resisting therapies for various kinds of tumors (17), including breast cancer, colorectal carcinoma, gastric cancer and glioblastoma multiforme (18-22), as well as hepatocellular carcinoma, miR-101 and miR-122 were downregulated in HCC cells and associated with resistance to chemotherapy or drug sensitization $(23,24)$. However, the deviant expression and the potential role of some miRNAs in hepatocellular carcinoma are under studied. Upregulation of miR-503 has been noted to increase the drug sensitivity $(2,13)$. Our data also demonstrated that miR-503 may improve the response of HCC cells to 5-FU.

Consequently, we distinguished the putative tumor suppressive function of miR-503 in human hepatocellular carcinoma cell lines. We initially looked at the mechanism of miR-503 on hepatocellular carcinoma cell drug sensitivity, and discovered that reinstatement of miR-503 in the hepatocellular carcinoma cell lines HepG2, BEL-7402 and SMMC-7721 substantially improved the sensitivity of hepatocellular carcinoma cells to 5-FU, via cell viability assays. The drug sensitivity function of miR-503 may be connected to the fact that miR-503 focuses on 3'-UTR of EIF4E mRNA, and prevents EIF4E expression in hepatocellular carcinoma cells. That was demonstrated in the present study in conjunction with the inhibition of cell proliferation. After transfection with miR-503, EIF4E expression was downregulated.

Epigenetic silencing of miRNAs using tumor limiting attributes is a common trademark of human tumors. Having demonstrated the critical function of miR-503 in limiting human hepatocellular carcinoma advancement, we aimed to reveal the potential gene effectors engaging in its activity. It is important to consider that miR-503 has demonstrated an ability to target multiple oncogenes and proliferation regulators (25-29), but surrounded by the miR-503 expected to target genes, we discovered that EIF4E acts as a crucial effector of miR-503. We revealed that miR-503 was able to substantially subdue the luciferase activity of Luc-EIF4E-3'-UTR by targeting the 3'-UTR of EIF4E mRNA, so we concentrated on EIF4E for additional examination.

In the present study, we determined that miR-503 was a possible prognostic marker for hepatocellular carcinoma, and discovered that miR-503 is extremely downregulated in human hepatocellular carcinoma tissues compared with normal liver tissues. Moreover, we also exposed that the upregulation of miR-503 prevents HCC cell proliferation and increases pharmaceutical sensitivity to some extent through modulating expression of EIF4E in hepatocellular carcinoma cells. The experimental data could offer a way to pinpoint the miR-503/ EIF4E interaction in a new therapeutic application to care for people suffering from hepatocellular carcinoma.

\section{Acknowledgements}

This study was funded by the National Natural Sciences Foundation of China (no. 81372579); the Health Department Medical Scientific Research Projects of Hunan Province (no. B2014-048); the Hunan Provincial Education Department (no. 13C832). We would like to thank LetPub (www.letpub.com) for its linguistic assistance during the preparation of this study.

\section{References}

1. Jemal A, Bray F, Center MM, Ferlay J, Ward E and Forman D: Global cancer statistics. CA Cancer J Clin 61: 69-90, 2011.

2. Qiu T, Zhou L, Wang T, Xu J, Wang J, Chen W, Zhou X, Huang Z, Zhu W, Shu Y, et al: miR-503 regulates the resistance of non-small cell lung cancer cells to cisplatin by targeting Bcl-2. Int J Mol Med 32: 593-598, 2013.

3. Fojo T: Multiple paths to a drug resistance phenotype: Mutations, translocations, deletions and amplification of coding genes or promoter regions, epigenetic changes and microRNAs. Drug Resist Updat 10: 59-67, 2007.

4. Glasspool RM, Teodoridis JM and Brown R: Epigenetics as a mechanism driving polygenic clinical drug resistance. $\mathrm{Br} \mathrm{J}$ Cancer 94: 1087-1092, 2006.

5. Sharma SV, Lee DY, Li B, Quinlan MP, Takahashi F, Maheswaran S, McDermott U, Azizian N, Zou L, Fischbach MA, et al: A chromatin-mediated reversible drug-tolerant state in cancer cell subpopulations. Cell 141: 69-80, 2010.

6. Lee RC, Feinbaum RL and Ambros V: The C.elegans heterochronic gene lin-4 encodes small RNAs with antisense complementarity to lin-14. Cell 75: 843-854, 1993.

7. Hobert O: Gene regulation by transcription factors and microRNAs. Science 319: 1785-1786, 2008.

8. Griffiths-Jones S, Saini HK, van Dongen S and Enright AJ: miRBase: Tools for microRNA genomics. Nucleic Acids Res 36: D154-D158, 2008.

9. Zhao JJ, Yang J, Lin J, Yao N, Zhu Y, Zheng J, Xu J, Cheng JQ, Lin JY and Ma X: Identification of miRNAs associated with tumorigenesis of retinoblastoma by miRNA microarray analysis. Childs Nerv Syst 25: 13-20, 2009.

10. Peng Y, Liu YM, Li LC, Wang LL and Wu XL: microRNA-503 inhibits gastric cancer cell grow th and epithelial-to-mesenchymal transition. Oncol Lett 7: 1233-1238, 2014.

11. Chong Y, Zhang J, Guo X, Li G, Zhang S, Li C, Jiao Z and Shao M: MicroRNA-503 acts as a tumor suppressor in osteosarcoma by targeting L1CAM. PLoS One 9: e114585, 2014. 
12. Zhang Y, Chen X, Lian H, Liu J, Zhou B, Han S, Peng B, Yin J, Liu W and He X: MicroRNA-503 acts as a tumor suppressor in glioblastoma for multiple antitumor effects by targeting IGF-1R. Oncol Rep 31: 1445-1452, 2014.

13. Wang T, Ge G, Ding Y, Zhou X, Huang Z, Zhu W, Shu Y and Liu P: miR-503 regulates cisplatin resistance of human gastric cancer cell lines by targeting IGF1R and BCL2. Chin Med J (Engl) 127: 2357-2362, 2014.

14. Sunavala-Dossabhoy G, Palaniyandi S, Clark C, Nathan CO, Abreo FW and Caldito G: Analysis of eIF4E and 4EBP1 mRNAs in head and neck cancer. Laryngoscope 121: 2136-2141, 2011.

15. Singh J, Jayaraj R, Baxi S, Mileva M, Curtin J and Thomas M: An Australian retrospective study to evaluate the prognostic role of $\mathrm{p} 53$ and eIF4E cancer markers in patients with head and neck squamous cell carcinoma (HNSCC): Study protocol. Asian Pac J Cancer Prev 14: 4717-4721, 2013.

16. Yin X, Kim RH, Sun G, Miller JK and Li BD: Overexpression of eukaryotic initiation factor $4 \mathrm{E}$ is correlated with increased risk for systemic dissemination in node-positive breast cancer patients. J Am Coll Surg 218: 663-671, 2014.

17. Garzon R, Marcucci G and Croce CM: Targeting microRNAs in cancer: Rationale, strategies and challenges. Nat Rev Drug Discov 9: 775-789, 2010.

18. Chen GQ, Zhao ZW, Zhou HY, Liu YJ and Yang HJ: Systematic analysis of microRNA involved in resistance of the MCF-7 human breast cancer cell to doxorubicin. Med Oncol 27: 406-415, 2010.

19. Li Y, Li W, Yang Y, Lu Y, He C, Hu G, Liu H, Chen J, He J and Yu H: MicroRNA-21 targets LRRFIP1 and contributes to VM-26 resistance in glioblastoma multiforme. Brain Res 1286: 13-18, 2009.

20. Xu K, Liang X, Cui D, Wu Y, Shi W and Liu J: miR-1915 inhibits Bcl-2 to modulate multidrug resistance by increasing drug-sensitivity in human colorectal carcinoma cells. Mol Carcinog 52: 70-78, 2013.

21. Xia L, Zhang D, Du R, Pan Y, Zhao L, Sun S, Hong L, Liu J and Fan D: miR-15b and miR-16 modulate multidrug resistance by targeting BCL2 in human gastric cancer cells. Int J Cancer 123 $372-379,2008$
22. Chen J, Tian W, Cai H, He H and Deng Y: Downregulation of microRNA-200c is associated with drug resistance in human breast cancer. Med Oncol 29: 2527-2534, 2012.

23. He H, Tian W, Chen H and Deng Y: MicroRNA-101 sensitizes hepatocellular carcinoma cells to doxorubicin-induced apoptosis via targeting Mcl-1. Mol Med Rep 13: 1923-1929, 2016.

24. Xu Y, Huang J, Ma L, Shan J, Shen J, Yang Z, Liu L, Luo Y, Yao C and Qian C: MicroRNA-122 confers sorafenib resistance to hepatocellular carcinoma cells by targeting IGF-1R to regulate RAS/RAF/ERK signaling pathways. Cancer Lett 371: 171-181, 2016.

25. Long J, Ou C, Xia H, Zhu Y and Liu D: MiR-503 inhibited cell proliferation of human breast cancer cells by suppressing CCND1 expression. Tumour Biol 36: 8697-8702, 2015.

26. Yang Y, Liu L, Zhang Y, Guan H, Wu J, Zhu X, Yuan J and Li M: MiR-503 targets PI3K p85 and IKK- $\beta$ and suppresses progression of non-small cell lung cancer. Int J Cancer 135: 1531-1542, 2014.

27. Xiao F, Zhang W, Chen L, Chen F, Xie H, Xing C, Yu X, Ding S, Chen K, Guo H, et al: MicroRNA-503 inhibits the G1/S transition by downregulating cyclin D3 and E2F3 in hepatocellular carcinoma. J Transl Med 11: 195, 2013.

28. Xu YY,Wu HJ,MaHD, Xu LP,Huo Y and Yin LR: MicroRNA-503 suppresses proliferation and cell-cycle progression of endometrioid endometrial cancer by negatively regulating cyclin D1. FEBS J 280: 3768-3779, 2013.

29. Forrest AR, Kanamori-Katayama M, Tomaru Y, Lassmann T, Ninomiya N, Takahashi Y, de Hoon MJ, Kubosaki A, Kaiho A, Suzuki M, et al: Induction of microRNAs, miR-155, miR-222, miR-424 and miR-503, promotes monocytic differentiation through combinatorial regulation. Leukemia 24: 460-466, 2010 . 